\title{
Upper limb involvement in cervical spondylosis
}

\author{
DOUGLAS G. PHILLIPS
}

\author{
From the Department of Neurological Surgery, Frenchay Hospital, Bristol
}

SYNOPSIS Analysis of 200 cases reveals that the two neurological syndromes, brachial neuritis and myelopathy, associated with cervical spondylosis are distinct with relatively little overlap. While upper limb motor and sensory loss are doubtless due to nerve root compression in cases of 'pure' brachial neuritis, they are more likely to be due to cord damage in cases with myelopathy (with spastic paraparesis of lower limbs). In either group of cases, neurological features in the upper limbs are not very helpful in localizing the level of significant intervertebral disc pathology. Contrast radiology (myelography and possibly discography) is a reliable guide judging by the excellent results obtained by anterior route (Cloward's) operation at specific disc levels in a series of cases with longstanding complaints unrelieved by conservative treatment. Pathological data provide a rational basis for interpretation of clinical observations and for surgical treatment.

A previous publication (Phillips, 1973) surveyed the results of surgical treatment of myelopathy with cervical spondylosis. As usual in such surveys, results were judged by relief of spastic paraparesis, the most persistent and serious feature of the illness and also the most easily measured. It was concluded, contrary to some previously held and still prevalent opinions, that myelopathy from compression of the cervical cord in this disorder is amenable to surgical treatment.

In contrast, it has long been considered that brachial neuritis associated with cervical spondylosis is a more benign condition, responding almost invariably to adequate conservative, nonsurgical treatment (Campbell and Phillips, 1960). Our experience in the last decade indicates that this concept needs revision. Moreover, study of the clinical features and pathogenesis of the condition reveals previously unsuspected problems.

\section{METHODS}

PATIENTS This study is based on the clinical and radiological findings and the results of treatment in consecutive series of 50 cases of cervical spondylosis with brachial neuritis-that is, without any involvement of the lower limbs indicating long tract compression in the spinal cord-and 151 cases of cervical spondylosis with myelopathy (of which 111 cases, up to 1970, were reported in 1973), treated as inpatients in the Bristol Neurosurgical Unit since 1961.

ANALYSIS Rather surprisingly, the two groups were more mutually exclusive than might have been expected. By definition, the brachial neuritis group had no long tract signs, the symptoms and signs being confined to the neck, shoulder girdle, and upper limbs, the predominant feature being pain in the upper limb or brachial neuralgia which was severe in most cases and present to some degree in all. In all but 10 cases there were objective signs of motor or sensory impairment in the upper limbs. In six of the 10 exceptions localized paraesthesiae in the upper limb suggested nerve root involvement.

While upper limb involvement was absent in only 12 of the myelopathy group, pain in the shoulder girdle or upper limb (brachial neuralgia) was present in only 25 cases. In nearly all of these, pain was a minor symptom but paraesthesiae, being present in 80 cases, were as common in this 'myelopathy' group as in the 'brachial neuritis' group. Objective motor and sensory impairment in the upper limbs was roughly comparable in the two groups-motor impairment in 113 cases and sensory of impairment in 60 cases of the myelopathy group, the corresponding numbers in the brachial neuritis group being 41 and 27 .

The age of onset of neurological disorder in the brachial neuritis group varied from 22 to 65 years 
(average 47.5 years). Onset of myelopathy varied between 23 and 80 years (average 57 years).

The average duration of symptoms before treatment in the neurosurgical unit was 2.7 years in the brachial neuritis group. Apart from two cases with a history of only 10 days and three weeks, the length of history varied from two months to 10 years (13 cases under one year, 11 cases one to two years, 26 cases over two years). The average duration of symptoms of myelopathy was 2.15 years, varying from one month to 16 years $(75$ cases under one year, 25 cases one to two years, 50 cases over two years).

The incidence of the significant levels of osteophyte and/or disc encroachment on the cervical spinal canal as judged by myelography (performed in all cases), and in a few cases by discography, was as indicated in Table 1.

A single level was judged significant in 25 of the brachial neuritis cases, and 60 of the myelopathies. In the others two or more levels were concerned (and treated, where operation was performed).

TABLE 1

INCIDENCE OF DISC INVOLVEMENT

\begin{tabular}{lrrrrc}
\hline & \multicolumn{5}{c}{ Disc level } \\
\cline { 2 - 6 } & $C 3 / 4$ & $4 / 5$ & $5 / 6$ & $6 / 7$ & $C 7 / T 1$ \\
\hline Brachial neuritis & 4 & 6 & 33 & 26 & 5 \\
Myelopathy & 50 & 52 & 91 & 51 & 3 \\
\hline
\end{tabular}

It is notable that in cases with myelopathy the spread of pathology, while maximal at the C5/6 intravertebral space, is evenly distributed at the two disc levels above and one below this level, with only three cases having a lesion demonstrable at the C7/T1 disc. With the 'pure' brachial neuritis the frequency of lesions at $\mathrm{C} 5 / 6$ disc space more closely resembled that at $\mathrm{C} 5 / 6$ space, while the incidence at the other affected levels from $\mathrm{C} 3$ to $\mathrm{T} 1$ vertebra was very much lower.

Motor signs were present in 41 cases of brachial neuritis and in 113 cases with myelopathy. In both groups, some degree of weakness and wasting of the intrinsic muscles of the hands was commonly present, whatever the level of the disc pathology. (This is a useful diagnostic feature when found with spastic paraparesis in the presence of cervical spondylosis.)

Particularly interesting in this study has been the segmental disc pathology in cases with weakness and wasting of the shoulder girdle and deltoid muscles.
In the brachial neuritis group, there were six such cases. In three of these, the abnormal disc levels were $\mathrm{C} 3 / 4,5 / 6$. In one case the highest level was $\mathrm{C} 4 / 5$ disc (C5/6 also being affected) and in two cases the affected levels were $\mathrm{C} 5 / 6$, and $\mathrm{C} 5 / 6,6 / 7$ discs. In another case, C3/4 was the only disc level involved, and weakness and wasting were confined to the hand, without arm or shoulder involvement.

The findings in the group where upper limb involvement was associated with myelopathy were different. Thus, in the 25 cases with weakness and wasting of shoulder girdle and deltoid muscles, in no less than 22 the $\mathrm{C} 3 / 4$ disc level was involved (though in 19 of these cases, lower levels were also affected). In contrast, only three cases had shoulder girdle and deltoid weakness and wasting where the $\mathrm{C} 3 / 4$ disc was not involved (in these the affected levels were $\mathrm{C} 4 / 5$ in two cases, and C5/6 in one).

In the brachial neuritis group pain involved the shoulder in 25 cases but in all of these it spread to the arm, and in nearly all to the hand as well. Pain in the fingers, however, occurred only with $\mathrm{C} 6 / 7$ and C7/T1 disc involvement. Distribution of paraesthesiae (present in 29 cases of brachial neuritis and 80 with myelopathy) was sometimes indicative of the pathological level; tingling of thumb and adjacent fingers occurred with C5/6 pathology, and of the ulnar two or three fingers with $\mathrm{C} 6 / 7$ pathology. The same applied to cutaneous sensory impairment in the hand when it was present ( 27 cases of brachial neuritis, 60 myelopathy) but the findings were not consistent or always easy to define.

Examination of the tendon reflexes most commonly showed that the biceps, triceps, and supinator jerks were all present and brisk. In the brachial neuritis group there was absence of isolated tendon jerks in only eight cases, and the pattern bore no particular relation to segmental distribution of the disc lesions. The findings were rather more consistent in the 19 cases with one or more absent tendon jerks in the myelopathy group. Here the biceps and supinator jerks were absent in ten cases where $\mathrm{C} 3 / 4,4 / 5$, and $5 / 6$ discs were affected. All these jerks were absent in one or other upper limb in six cases with disc pathology from $\mathrm{C} 4 / 5$ to $\mathrm{C} 6 / 7$; the triceps jerk alone was absent in only two cases, $\mathrm{C} 5 / 6$ and $\mathrm{C} 6 / 7$ being the levels involved.

SELECTION OF CASES FOR SURGICAL TREATMENT Virtually all cases resistant to conservative measures have had operative treatment. As is clear from the statement on average duration of symptoms before surgical treatment, most patients have had prolonged disability. They have had various treatments (most by various collars, some by traction) with limited benefit. Those with shorter histories have often had 
more severe or more rapid progression of symptoms and signs. The greater number of cases with myelopathy who were treated as inpatients in a surgical unit is a reflection of the more serious implications of cord compression and the fact that brachial neuritis is more responsive to adequate immobilization of the neck. A modified light Minerva plaster collar is still found useful, being more effective than any short collar. Even so, with experience of the effectiveness of operation in persistent or relapsing brachial neuritis, as with myelopathy, there is less hesitation in advising it.

Myelography has been performed in all cases of this series preoperatively, and discography in some. Other causes of spinal compression must be excluded, particularly where operation, as with nearly all of our cases, is by an anterior approach without the extensive exposure of a laminectomy. The significant level, or levels, of compression by osteophytes and protruding discs as judged by preliminary contrast radiography must be identified. If there is reasonable possibility of more than one level being involved, two and occasionally three levels are dealt with at one operation.

Cloward's operation has been the operation of choice in nearly all cases of brachial neuritis, and in most cases with myelopathy. This operation by an anterior approach involves a half inch $(1.2 \mathrm{~cm})$ drill hole at the level of the affected disc(s), with careful and complete removal of all compressing elements (osteophytes and discs, bone, cartilage, and fibrous tissue) projecting into the spinal canal in front of the theca. This cannot be done through a posterior laminectomy approach, and the Cloward's operation gives wider access than the Smith-Robinson method, which means working through the disc space only. A bone graft in the drill hole ensures immobilization and fusion at the treated level(s), without necessity for further collars.

Tables 2 and 3 summarize the results of this operation in all cases with two or more years followup performed at the level or levels identified by radiology.

It will be seen that relief of pain was very satisfactory and lasting in nearly all cases. Very often the patients volunteered immediately after operation that the pain in the upper limb was gone, and that they could use their hands better. However, some sensory and motor loss was liable to remain, especially if very marked or longstanding.

\section{DISCUSSION}

The high incidence of disorder of $\mathrm{C} 5 / 6$ and to slightly lesser degree of C6/7 intervertebral discs
TABLE 2

RESULTS OF CLOWARD'S OPERATION FOR BRACHIAL NEURITIS (29 CASES) 1961-72 (TWO-10 YEARS' FOLLOW-UP)

\begin{tabular}{lccc}
\hline & Pain & Motor loss & Sensory loss \\
\hline Relief & 25 & 13 & 9 \\
Totals & $29^{*}$ & 24 & 18 \\
\hline
\end{tabular}

* One no relief, three relapsed within two years.

TABLE 3

RESULTS OF CLOWARD'S OPERATION IN CASES OF MYELOPATHY WITH UPPER LIMB INVOLVEMENT (76 CASES) 1961-72 (TWO TO 10 YEARS FOLLOW-UP)

\begin{tabular}{lrcc}
\hline & Pain & Motor loss & Sensory loss \\
\hline $\begin{array}{l}\text { Relief } \\
\text { (in upper limbs) }\end{array}$ & 9 & 39 & 15 \\
Totals & 10 & 61 & 32 \\
\hline
\end{tabular}

NB. Relief of pain here implies complete or virtually complete relief with no disability. Relief of motor or sensory loss in many cases was partial, but usually enough to relieve disability.

in cervical spondylosis is well known. It is not easy to explain the very low incidence of upper limb involvement with disc and vertebral body pathology at the levels of $\mathrm{C} 3 / 4$ and $\mathrm{C} 4 / 5$ intervertebral spaces where there is no long tract involvement. It is customarily postulated that the pathogenesis differs in the two groupsnamely, that cord compression must be due to 'central' disc prolapse and/or osteophytes, while brachial neuritis is attributed to more lateral compression involving the nerve root. The high incidence of upper limb involvement associated with demonstrable pathology at $\mathrm{C} 3 / 4$ and $\mathrm{C} 4 / 5$ disc spaces in the myelopathy group, suggests that in these cases at least upper limb involvement is more likely to be associated with

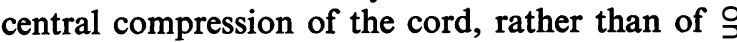
nerve roots at or near the intervertebral foramina.

This hypothesis is further supported by comparison of the presenting neurological signs (motor, sensory, and reflex) in the upper limbs in each group. If wasting and weakness of the hand is particularly pronounced, it suggests involvement of $\mathrm{C} 8$ or $\mathrm{T} 1$ anterior nerve roots (or associated anterior horn cells). Marked weak- 
ness and wasting of the triceps muscle suggests C7 segmental involvement. If weakness and wasting of the biceps muscle is the prominent feature, involvement of $\mathrm{C} 6$ myotome is suggested. However, clear differentiation is often not possible, and, even when it is, is not reliable as an indication of the precise level of the lesion. Pre- and post-fixation of the brachial plexus, and multiple disc pathology are two factors which could be implicated.

One would expect shoulder girdle and deltoid weakness and wasting to result in cases of brachial neuritis from pressure on the 5th cervical motor root-that is, at the level of $\mathrm{C} 4 / 5$ intervertebral disc, where the 5 th root passes through the intervertebral foramen. The actual findings did not correspond, as in only one such case did the $\mathrm{C} 4 / 5$ interspace appear pathological, the affected discs being above or below this level in the other five cases.

The relationships were more striking in the myelopathy group, in that shoulder girdle and deltoid wasting was virtually confined to cases where the C3/4 disc space was affected. This might be explained on the assumption that in this group the motor loss was from anterior horn cell compression by a 'central' disc or osteophyte protrusion, rather than from root compression. Even so, it was surprising that the level of disc pathology was so high, and that root compression would appear not to be very commonly associated with cord compression at the same level. The rarity of upper limb pain of any severity in this group with myelopathy would be in accord with this conclusion, though as previously noted, paraesthesiae in the upper limb were of equal incidence in both groups.

Sensory phenomena generally (pain, paraesthesiae, and cutaneous sensory loss), and tendon reflexes, like wasting and weakness (apart from involvement of the shoulder girdle in cases with myelopathy indicated by lower limb involvement) were quite unhelpful in determining significant levels of disc pathology.

Thus, we early came to the conclusion, reinforced by long experience, that contrary to the statements of many physicians on this topicbut in agreement with Brain et al. (1952)neurological findings are of extremely limited use in assessment of the precise level of cord and root involvement, and may be misleading. We have therefore relied on careful radiological studies (always with myelography, and sometimes with discography and tomography). This policy has been supported by the results of surgical treatment over the past 13 years.

Detailed necropsy studies are rare in this condition and, by its nature, the recorded findings in myelopathy, and in advanced cases, are more plentiful than in cases of brachial neuritis where the disorder affects the upper limbs alone. However, most accounts indicate that in myelopathy at least, the neurological disorder is due to direct compression of the nerve elements, often at one specific level-and that diffuse vascular pathology is not likely to be so important. 'Direct' injury of the spinal cord is indicated by destruction or damage identifiable histologically in the grey matter as well as white matter in the cord at the affected level. Above this there will be degeneration in ascending tracts, and below, in descending tracts in the white matter. Hughes (1966) pointed out that at necropsy all cases of cervical spondylosis with myelopathy show deformity of the cord due to indentations, though occasionally indentations may occur without clinical or pathological evidence of myelopathy. Payne and Spillane (1957) concluded that in some cases postmortem indentations were artefacts. (Also, one cannot rely on plain radiography of the cervical spine to identify the significant levels of disc pathology responsible for cord or nerve root damage. Large soft tissue projections require demonstration by myelography.) It does seem, however, that (1) there is usually a single level of severe direct cord damage (even if there are multiple levels of disc pathology, one is usually identifiable as the largest; in cases of doubt one may select two levels as possibly causing direct cord damage, sometimes three); (2) this pathological segmental level is usually one 'below' the disc level identified as the most pathological, in cases of myelopathy-for example, C3/4 disc pathology is associated with C5 segmental neurological abnormality.

Payne and Spillane (1957) commented on the relationship of spinal cord segments and nerve roots to cervical disc levels. On the basis of 
anatomical studies, they found that the cervical nerve roots did not pass out horizontally, as often stated, but that

'roots below $\mathrm{C} 3$ pass downwards with increasing obliquity to reach their respective intervertebral foramina. At and below the C5 segment, this obliquity is sufficient for at least part of the root exit zone (i.e. in the cord) to lie at one disc higher than the foramen through which the radicular nerve leaves the spinal canal. For example, $\mathrm{C} 7$ segment lies opposite $\mathrm{C} 5 / 6$ disc, whereas the $\mathrm{C} 7$ radicular nerve crosses the lateral aspect of $\mathrm{C6} / 7$ disc'.

These considerations are illustrated by the findings of postmortem studies listed in Table 4.

Wallerian degeneration or deficiency in axons of nerve roots was found in only one (case 1) of the cases described by Brain et al. (1952), the others showing flattening of roots without histological changes. In Mair and Druckman's (1953)

TABLE 4

POSTMORTEM FINDINGS OF LOCAL CORD DAMAGE

\begin{tabular}{llcc}
\hline Authors & $\begin{array}{c}\text { Single level } \\
\text { direct cord } \\
\text { damage } \\
\text { (case no.) }\end{array}$ & $\begin{array}{c}\text { Two } \\
\text { segmental } \\
\text { levels } \\
\text { involved } \\
\text { (case no.) }\end{array}$ & $\begin{array}{c}\text { Segmental } \\
\text { cord destruc- } \\
\text { tion described } \\
\text { as one segment } \\
\text { below worst } \\
\text { disc level } \\
\text { (case no.) }\end{array}$ \\
\hline $\begin{array}{l}\text { Bedford } \text { et al. }(1952) \\
\text { (one case) }\end{array}$ & 1 & \\
$\begin{array}{c}\text { Brain } \text { et al. (1952) } \\
\text { (six cases) }\end{array}$ & $2,3^{*}, 4^{*}, 5^{*}, 6^{*}$ & $3,4,5,6$ \\
$\begin{array}{l}\text { Mair and Druckman } \\
\text { (1953) (four cases) }\end{array}$ & $1,2^{*}, 3^{*}, 4$ & $1,2,3$ \\
$\begin{array}{l}\text { Wilkinson (1960) } \\
\text { (four cases in detail } \\
\text { excluding cases } 5\end{array}$ & $1,2^{*}, 4^{*}$ & 3 & 3 \\
$\begin{array}{l}\text { and 6 already de- } \\
\text { scribed by Brain } \text { et } \\
\text { al. (1952) as cases } \\
2 \text { and 4) }\end{array}$ & & \\
\hline
\end{tabular}

* Multiple dis srotrusions, direct cord damage opposite largest.

† For example, segmental cord damage described as C5, opposite C3-4 disc protrusion.

series, damage to posterior root fibres in the most affected segment in the posterior root entry zone occurred in case 1 only. The anterior nerve roots were deficient in axons of the affected segment in three cases. The corresponding anterior horns showed loss of nerve cells.
The available pathological data are consistent with the clinical observations previously described and do provide a rational basis for searching out and identifying by radiological means the most significant intraspinal projections at cervical intervertebral disc levels. These bony and soft tissue projections may then be removed radically. Obviously the amount of neurological recovery that is possible will depend on the degree of cord and nerve root damage, which in turn depends on the severity of the compression and its duration-and can be assessed, in the absence of necropsy, only by clinical findings. In cases where severe neurological disorder results from a spinal 'extension' injury the initial abrupt damage may be permanent (as with any case of traumatic paraplegia), or there may be more or less spontaneous recovery from cord contusion. In cases where there is no history of trauma (or where trauma is only a relatively minor incident preceding or following the onset of progressive symptoms) experience has shown that, provided the case is not too advanced, prompt, progressive, and lasting relief, to some degree, is likely to follow adequate removal of the compressing agent whether it affects the cord or the nerve roots, of both.

\section{REFERENCES}

Bedford, P. D., Bosanquet, F. D., and Russell, W. R. (1952). Degeneration of the spinal cord associated with cervical spondylosis. Lancet, 2 , 55-59.

Brain, W. R., Northfield, D. W. C., and Wilkinson, M. (1952). The neurological manifestations of cervical spondylosis. Brain, 75, 187-225.

Campbell, A. M. G., and Phillips, D. G. (1960). Cervical disk lesions with neurological disorder. British Medical Journal, 2, 481-485.

Hughes, J. T. (1966). Pathology of the Spinal Cord. LloydLuke: London.

Mair, W. G. P., and Druckman, R. (1953). The pathology of spinal cord lesions and their relation to the clinical features in protrusion of the cervical intervertebral discs. Brain, 76, 70-91.

Payne, E. E., and Spillane, J. D. (1957). The cervical spine. An anatomico-pathological study of 70 specimens with particular reference to the problem of cervical spondylosis. Brain, 80, 571-596.

Phillips, D. G. (1973). Surgical treatment of myelopathy with cervical spondylosis. Journal of Neurology, Neurosurgery, and Psychiatry, 36, 879-884.

Wilkinson, M. (1960). The morbid anatomy of cervical spondylosis and myelopathy. Brain, 83, 589-617. 